\title{
Efficacy Comparison of Ureteroscopic Holmium Laser Lithotripsy and Pneumatic Lithotripsy on Ureteral Calculi of the Elderly
}

\author{
Li He Wang \\ No.2 Affiliated hospital of Qiqihaer Medical University, Qiqihaer, 161000,China \\ email: wanglihe5672@126.com
}

Keywords: Holmium laser lithotripsy; Pneumatic lithotripsy; Ureteral calculi; Comparative efficacy

\begin{abstract}
Department of Urology in Zunyi County People's Hospital from 2008 to September 2010, were comparatively analyzed. calculi breaking success rate, calculi clearance rate, operation time, hospital stay and other aspect of the two treatment methods were observed. The results showed that one-time success rate of LL group was higher than that of PL group with significant difference; Clearance rate of LL group was significantly higher than that of PL group with significant difference. Operation time of LL group was significantly less than that of PL group with significant difference; comparative difference of hospital stay had no statistical significance. Comparative analysis showed that LL group was better than PL group in three aspects of one-time calculi breaking success rate, calculi clearance rate, operation time, etc., which is ideal calculi breaking means for treating ureteral calculi for the elderly.
\end{abstract}

\section{Introduction}

Ureteral calculi accounts for $33 \%$ to $54 \%$ of uri-nary calculis. More than $90 \%$ of ureteral calculi is discovered from kidney. Its composition is similar with kidney calculis, and the shapes are mostly based on circle and date pit. Clinical manifestations are related with calculi size, activity, obstruction degree and infection. Most patients have symptoms main including pain and hematuria. Ureteral calculi can be accompanied by secondary and concurrent obstruction, infection, polyps or tumors, thereby leading to hydronephrosis, renal parenchymal oppression thinning, kidney function damage and even function loss, etc. Therefore, patients can not recover as quickly as possible by correct treatment, and occurrence of various complications also can be prevented. Currently, separate treatment of ureteral calculi by ureteroscopic holmium laser lithotripsy and pneumatic lithotripsy is more studied. However, there is less literature on comparative study on the two surgery methods with single comparative indicators [1,2], such as one-time calculi breaking success rate, complication, etc. Sample results are not statistically tested in some literature[3,4].

Population aging is further exacerbated with life quality improvement of people in China. Elderly pa-tients with ureteral calculi is also growing year by year. However, patients are always accompanied with some underlying diseases, such as hypertension, diabetes, coronary heart disease, etc., thereby increasing treatment difficulty and risk. Patients with multiple diseases have poor tolerance ability especially. Risks of surgery and anesthesia are prominently increased. Postoperative recovery was slow, and it is prone to produce various complications. It is particularly important to manage peri-operative period sufficiently. Ureteroscopic holmium laser lithotripsy, as a mature technology, is widely used in clinical purpose with precise treatment effect and less risk. It is always best choice for treating ureteral calculi in middle and low sections. However, treatment of ureteral calculi for the elderly is rarely reported with insufficient systematic analysis. Holmium laser (holmium: YAG laser) is the latest solid pulse laser in surgical operation at present. The absorption coefficient of water is large at $2100 \mathrm{~nm}$ wavelength. Human tissues are mainly composed of water. Therefore, laser energy can be transmitted to calculi when calculi is broken by holmium laser lithotripsy (laser lithotripsy, LL), thereby leading to fragmentation of calculi. A lot of energy is absorbed by water, thereby avoiding injury on surrounding tissues. LL is applied late, but it has been widely accepted compared with pneumatic lithotripsy (pnumatic lithotripsy, PL). Both lithotripsy methods are widely used for the 
clinical treatment of ureteral calculi. It is reported that the safety and efficacy of LL were significantly improved compared with PL. However, comparison reports of both lithotripsy methods on ureteral calculi treatment for the elderly are rare. In the study, therapy of treating ureteral calculi for the elderly by LL and PL in our hospital is retrospectively ana-lyzed[5].

\section{Data and Methods}

Data. 77 elderly patients with ureteral calculi, treated by Department of Urology in Zunyi County People's Hospital from March 2008 to September 2010 were retrospectively analyzed, including 44 male patients and 31 female patients. They were divided into holmium laser surgery (LL) group and pneumatic lithotripsy surgery (PL) group according to different operation methods. All patients underwent kidney ureter and bladder (KUB), intravenousurography (IVU), ultrasound B or CT for clear diagnosis before operation. Case selection criteria included the follows: age $>60$ years; unilateral ureteral calculi, X-ray development and no combination of renal calculus requiring surgical treatment. It was confirmed that there was no ureter deformity or twist according to venography examination. In surgery, open operation was not included in the study group if ureteroscopy can not enter due to stricture of uretero-vesical orifice. LL group was composed of 40 patients, including 25 males and 15 females with mean age of $(65.15 \pm 10.21)$ years. 13 cases suffered from upper ureteral calculi, 6 cases suffered from middle ureteral calculi, and 21 cases suffered from lower ureteral calculi. The maximum diameter of calculi was $20 \mathrm{~mm}$, and the average size was $(11.18 \pm 1.12) \mathrm{mm}$. PL group was composed of 35 patients, including 20 males and 15 females with mean age of $(64.35 \pm 11.10)$ years. 10 cases suffered from upper ureteral calculi, 5 cases suffered from middle ureteral calculi, and 20 cases suffered from lower ureteral calculi. Maximum diameter of calculi was $15 \mathrm{~mm}$, the average size was $(10.02 \pm 167) \mathrm{mm}$. General data of the two groups was compared, and the difference had no statistical significance $(\mathrm{P}>0.05)$. Please see Table 1.

Tab 1 General Data Comparison of Patients in LL Group and PL Group

\begin{tabular}{|c|c|c|c|c|c|c|c|c|}
\hline \multirow{2}{*}{ Group } & \multirow{2}{*}{$\mathrm{n}$} & \multicolumn{2}{|c|}{ Gender } & \multirow{2}{*}{$\begin{array}{l}\text { Average } \\
\text { Age } \\
\text { (Year) }\end{array}$} & \multicolumn{3}{|c|}{ Calculi Position } & \multirow{2}{*}{$\begin{array}{l}\text { Calculi Size } \\
\text { (mm) }\end{array}$} \\
\hline & & Mae & Female & & Upper & Middle & Lower & \\
\hline LL Grc & 40 & 24 & 15 & $\begin{array}{c}65.15 \pm 10.2 \\
1\end{array}$ & 13 & 6 & 21 & $11.18 \pm$ \\
\hline PL Group & 35 & 20 & 15 & $64.35 \pm 11.10$ & 10 & 5 & 20 & $10.02 \pm 1.67$ \\
\hline
\end{tabular}

Method. Patient part with lithotomy underwent epidural anesthesia. F8/9.8 ureteroscopy produced by Germany WOLF Company was adopted, which was fed into bladder through urethra. After ureteral orifice on the affected side was discovered, PC guide wire was inserted into ureter of the affected size through ureteroscopy hole. Ureteroscopy was used for picking up the ureteral orifice close to the ureteral orifice, the ureter was rotated obliquely, which was slowly inserted into the ureter slowly under the guidance of guide wire, the Ureteroscopy was rotated to the original position. Extended part of the ureteroscopy in the conduit should not be too long, thereby avoiding pushing the calculi. Meanwhile, infusion fluid should be reduced, thereby avoiding calculi upward movement or renal calculi reflux due to high water pressure. The infusion fluid volume and pressure were adjusted to the level that the ureter mucosa can be clearly observed, the ureteroscopy was fed slowly under direct vision. Ureteral mucosa was observed for observing whether there were polyp, stenosis, bleeding and other conditions or not. The ureteroscopy was slowly pushed to calculi position. LL group adopted holmium laser machine produced by U.S. LUMENIS INC. After calculi was discovered, optical fiber was fed directly outside the calculi (power was set to 10-16 W). calculi was broken by 'nibbling' or 'insect biting' approach under direct vision according to calculi condition. The calculi was broken to be less than $3 \mathrm{~mm}$. Then calculi basket or calculi forceps were used for removing the calculi; Patients associated with ureteral polyps underwent laser removal of polyps firstly followed by calculi breaking. PL group adopts Swiss EWS pneumatic lithotripsy machine and 
hydraulic infusion pump. After the calculi was clearly observed, $1.2 \mathrm{~mm}$ pneumatic lithotripsy probe was fed from ureter operating chamber directly to the calculi. Air pressure was set to 1.5-2.0 atm. Calculi was broken to less than $3 \mathrm{~mm}$ in continuous pulse manner. Larger calculi was removed by calculi forceps. Patient associated with polyps should be burned by laser or the polyps should be sheared by calculi forceps after calculi breaking. Double $\mathrm{J}$ tube $3-4 \mathrm{w}$ was left for all patients after calculi breaking, thereby calculi chips can be removed and residue calculi can be processed. KUP plain film can be reviewed 3-4w after surgery, and doctor should determine to remove double $\mathrm{J}$ tube or not according to calculi residue condition.

Indicator Observation. On-time calculi breaking success rate, calculi clearance rate, surgery time, post-operation hospital stay, adverse effect and intraoperative complications (ureter injury and ureteral perforation) of the two groups are observed. KUB plain film is reviewed 3 to 4 weeks after operation. Calculi clearance depends on no calculi shadow in KUB examination.

Statistical Analysis. SPSS13.0 x \pm statistical software was applied, measurement data was expressed with $t$, test was adopted for difference comparison between the two groups, paired t test was adopted for front-rear com-parison in the same group. Measurement data was tested by $\chi 2$.

\section{Results and Discussion}

Results. There were no serious complications of ureteral perforation, tear and fever, obstructive renal abscess, etc. in the two groups. Calculi of one patient in LL group was moved to renal pelvis. ESWL was imple-mented after double $\mathrm{J}$ tube was reserved. 3 cases suffered from ureter mucosal bleeding during calculi breaking. Calculi of 4 patients in PL group was moved upwards to renal pelvis, ESWL was implemented after double $\mathrm{J}$ tube was reserved. 12 cases suffered from ureter mucosal bleeding during calculi breaking. One-time calculi breaking success rate of LL group was higher than that of PL group. The difference was statistically significant $(\mathrm{P}<0.05)$. KUB plain films were reviewed for the two groups $3 \mathrm{w}$ after operation. Calculi clearance rate of LL group was prominently higher than that of PL group. The difference was statistically significant $(\mathrm{P}<0.05)$. LL group operative time was significantly less than that of PL group. The difference was statistically significant $(\mathrm{P}<0$ 05.). In addition, hospital stay for the two groups was compared, and the difference was not statistically significant $(\mathrm{P}>0.05)$. Please see Table 2.

Tab 2 Comparison of Operation Conditions in The Two Groups

\begin{tabular}{ccc}
\hline Group & LL Group & PL Group \\
\hline $\mathrm{n}$ & 40 & 35 \\
One-time calculi Breaking Success rate & $95.17 \%$ & $81.03 \%$ \\
Calculi clearance rate & $98.40 \%$ & $82.18 \%$ \\
Operation Time (min) & $20.04 \pm 5.00$ & $28.13 \pm 6.45$ \\
Hospital Stay (d) & $6.78 \pm 1.05$ & $6.14 \pm 1.45$ \\
\hline
\end{tabular}

Discussion. There are various methods for treatment of ureteral calculi, such as extracorporeal shock wave therapy, lithotripsy, ureterolithotomy laparoscopic surgery and open surgery. Plan should be selected according to calculi size, quantity, shape, residence time, renal function as well as hospital circumstances (such as equipment, surgical personnel experience and technology, etc.). In recent years, application of open surgery in ureteral calculi is greatly reduced with development of endovascular technique. The author compared two endovascular techniques of LL and PL. Effect of LL group was better than that of PL group with higher efficiency. PL group adopted mechanical energy with no heat, no heat damage, no current, and extremely little heat release. Since the maximum impact magnitude of the elastomer is only $2 \mathrm{~mm}$, mucosal injury caused by edema and hemorrhage was very slight and transient. Acute injuries include edema, hemorrhage and mucosal shedding. However pathology and $\mathrm{X}$-ray show that the operation has no adverse effect. Pneumatic litho-tripsy is safe, effective and economical. However, calculi migration can be produced in the calculi breaking process. LL belongs to pulse launch, launch time is very short $(0.25 \mathrm{~ms})$, the instantaneous power is up 
to $10 \mathrm{~kW}$, calculi with various compositions and various densities can be broken without ureteral mucosal damage.

Two treatment methods were compared, the au-thor found that one-time calculi breaking rate of LL group is $95.17 \%$, which is significantly higher than $81.03 \%$ in PL group; LL group has shorter operative time, and two-month clearance rate is high. Reasons are analyzed, the values may be related with performance characteristics of holmium laser: YAG laser can emit high-energy pulsed solid-state laser, laser instantaneous peak power was up to $10 \mathrm{~kW}$, calculi can be easily crushed into tiny particles, which can be conveniently excluded; holmium laser can be used for stripping out calculi block embedded in polyp. Pneumatic lithotripsy has worse efficacy on the disease. Holmium laser can be used for treating polyps, ureteral stenosis, bleeding, etc. during lithotripsy. Calculi predisposing factors can be removed, factors unbeneficial for calculi removal can be eliminated, and calculi removal efficiency can be improved. Feeding of ureteroscopy is affected by ureteral polyps in PL group. Calculi or chips are usually embedded in polyps. It is difficult to further break calculi. After the polyps is cut or vaporized in LL group, ureteroscopy can be fed. 40 cases ac-companied by polyps in the group can be vaporized and cut, calculi is broken successfully, it is obvious that LL group is better than PL group in the aspect of treating calculi with polyps embedded into ureteral mucosa.

When surgery form is selected for lithotripsy, the calculi diameter should be less than $1 \mathrm{~cm}$, PL can be adopted for patient with calculi residence time in the ureter less than six weeks since the calculi residence time is short, polyps can be formed since the calculi is not embedded easily. LL can be applied for patient that calculi diameter is greater than $1 \mathrm{~cm}$, the residence time is more than six weeks, and polyps is available. Patient with longer disease duration and polyps, should adopt cutting and vaporization treatment, for example, polyps should be processed through cutting, vaporization, etc. Calculi can be broken from edge of calculi, namely top end of optical fiber can reach side of calculi, a lacuna can be produced by on the surface of edge for breaking calculi. Top end of optical fiber is repeatedly moved for expanding calculi breaking cavity, which is called 'inset biting' calculi breaking. The whole calculi can be broken into several large blocks from the center of calculi, then several large blocks can be broken again, more time is spent, the chips can be flushed out of vision or flushed into kidney by flowing water.

Double-J tube placement after surgery is still con-troversial. The author suggests to place the pipe ac-cording to experience. The Ureteroscopy process operation, mucosa suffers from edema, hemorrhage or injury at varying degrees. Some calculi chips are gathered together or form 'calculi street', thereby causing ureter obstruction, infection or renal dysfunction, double $\mathrm{J}$ pipe has the function of supporting ureteral and urine drainage. Small calculi can be moved downwards along double-J pipe. Therefore, double-J pipe can be reserved and the can be removed one to four weeks.

\section{Conclusion}

The paper shows that HL has the following features compared with PL:

A. One-time surgery calculi breaking success rate of holmium laser lithotripsy is higher.

B. Operative time of holmium laser lithotripsy is shorter.

C. Hospital stay and postoperative calculi clear-ance rate of lithotripsy in the two groups.

D. Postoperative fever incidence is lower in hol-mium laser group.

E. Intraoperative ureteral mucosa avulsion inci-dence, intraoperative ureteral perforation incidence and postoperative sustained hemorrhage incidence have no significant difference in the two groups.

In summary, ureteroscopic holmium laser lithotripsy and pneumatic lithotripsy are excellent methods for treating ureteral calculi. However, holmium laser is characterized by smaller trauma, higher efficiency and faster postoperative recovery of patient. However, they also can be used for processing ureteral calculi accompanied problems, such as polyps, narrow ring, etc. with unique advantages, and they are worth the clinical promoting. 


\section{References}

[1] QIN Bin, HUANG Xiang-hua, LI Zhang-zan, et al. omparison of holmium laser and pneumatic lithotripsy in managing ureteral calculi by ureteroscope. Chinese Journal of Endourology(Electronic Edition), 2012, 06(1):38-41.

[2] LIU Xuechao, YANG Ying , ZHANG Jianggen, et al. Comparative study of pneumatic lithotripsy and holmium laser lithotripsy for ureteral stones. Journal of Central South University(Medical Science), 2005;30(5):601-603.

[3] WANG Lei, LIU Xiuheng. Efficacy of Holmium laser ureterlithotripsy and pneumatic lithotripsy for upper ureteral calculi: a Meta analysis. China Medical Herald. 2013, 10(18):30-32.

[4] YANG Zesong, YE Liefu, ZHUANG Huiqiang, et al. Holmium:YAG Laser Lithotripsy Versus Pneumatic lithotripsy for Upper Ureteral Calculi. Journal of Clinical Urology. 2010, 25(1):16-18.

[5] TONG Moze, LIU Jianhui, Tao Hanshou, et al. A comparative study of holmium laser lithotripsy and pneumatic lithotripsy for ureteral stones. 